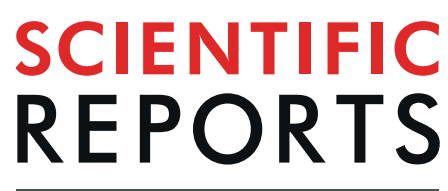

natureresearch

Check for updates

\title{
A Cell-based Screen in Actinomyces oris to Identify Sortase Inhibitors
}

\author{
Jason E. Gosschalk ${ }^{1,2}$, Chungyu Chang ${ }^{3}$, Christopher K. Sue ${ }^{1,2}$, Sara D. Siegel ${ }^{4}$, \\ Chenggang $\mathrm{Wu}^{4}$, Michele D. Kattke ${ }^{1,2}$, Sung Wook Yi ${ }^{1}$, Robert Damoiseaux ${ }^{5,6}$, \\ Michael E. Jung ${ }^{1,7}$, Hung Ton-That ${ }^{3,5} \otimes$ \& Robert T. Clubb ${ }^{1,2,7}$
}

Sortase enzymes are attractive antivirulence drug targets that attach virulence factors to the surface of Staphylococcus aureus and other medically significant bacterial pathogens. Prior efforts to discover a useful sortase inhibitor have relied upon an in vitro activity assay in which the enzyme is removed from its native site on the bacterial surface and truncated to improve solubility. To discover inhibitors that are effective in inactivating sortases in vivo, we developed and implemented a novel cell-based screen using Actinomyces oris, a key colonizer in the development of oral biofilms. A. oris is unique because it exhibits sortase-dependent growth in cell culture, providing a robust phenotype for high throughput screening (HTS). Three molecules representing two unique scaffolds were discovered by HTS and disrupt surface protein display in intact cells and inhibit enzyme activity in vitro. This represents the first HTS for sortase inhibitors that relies on the simple metric of cellular growth and suggests that $A$. oris may be a useful platform for discovery efforts targeting sortase.

Proteins displayed on the surface of bacterial pathogens play critical roles in the infection process by promoting bacterial adhesion to host tissues, acquisition of essential nutrients, evasion and suppression of the immune response and host-cell entry ${ }^{1,2}$. Gram-positive bacteria display virulence factors using sortases, cysteine transpeptidase enzymes that covalently attach proteins to peptidoglycan precursors or assemble pili ${ }^{3-6}$. Sortase enzymes are potential drug targets as they are required for the virulence of methicillin-resistant Staphylococcus aureus (MRSA), which causes a wide range of life-threatening diseases, such as pneumonia, meningitis, osteomyelitis, endocarditis, toxic shock syndrome, bacteremia, and sepsis ${ }^{7}$. These infections are major health concerns as they are estimated to cause 16,485 fatalities in the United States each year ${ }^{8}$. Sortase enzymes also contribute to the virulence of other clinically important pathogens, including among others: Enterococcus faecalis, Listeria monocytogenes, Bacillus anthracis, Streptococcus pyogenes and Streptococcus pneumoniae ${ }^{9}$. Thus, small-molecule inhibitors of sortase enzymes may prove to be useful antimicrobial agents to treat infections caused by MRSA and other bacterial pathogens.

The sortase enzyme in S. aureus ( ${ }^{\text {Sa SrtA) }}$ has been characterized to the greatest extent ${ }^{3-6}$. It recognizes protein substrates that harbor a C-terminal cell wall sorting signal (CWSS), which is comprised of a conserved LPXTG-type motif (where X denotes any amino acid), followed by a hydrophobic transmembrane segment and positively charged residues. The membrane-bound ${ }^{\mathrm{S}}$ SrtA covalently attaches protein substrates to the peptidoglycan via a transpeptidation reaction by cleaving the LPXTG motif between the Gly and Thr residues and joining the cleaved LPXT to the cross-bridge peptide of lipid II, a peptidoglycan precursor. Catalysis occurs through a ping-pong mechanism that is initiated when the active-site cysteine nucleophile attacks the backbone carbonyl carbon of the threonine residue within the LPXTG motif, breaking the threonine-glycine peptide bond to create a thioacyl-linked sortase-protein complex ${ }^{10-13}$. The protein-lipid II product of the sortase catalyzed reaction is formed when the thioacyl substrate-enzyme intermediate is resolved by the amino group within lipid II. Cell wall synthesis reactions then incorporate the protein-lipid II product into the peptidoglycan, displaying the protein on the microbial surface. Over 3,100 species of bacteria contain genes encoding for sortases related to ${ }^{\text {Sa }} \mathrm{SrtA}^{14,15}$,

\footnotetext{
${ }^{1}$ Department of Chemistry and Biochemistry, University of California, Los Angeles, USA. ${ }^{2}$ UCLA-DOE Institute of Genomics and Proteomics, University of California, Los Angeles, USA. ${ }^{3}$ Division of Oral Biology and Medicine, University of California, Los Angeles, USA. ${ }^{4}$ Department of Microbiology and Molecular Genetics, University of Texas Health Science Center, Houston, TX, USA. ${ }^{5}$ Department of Molecular and Medicinal Pharmacology, University of California, Los Angeles, USA. ${ }^{6}$ California NanoSystems Institute, University of California, Los Angeles, USA. ${ }^{7}$ Molecular Biology Institute, University of California, Los Angeles, 611 Charles Young Drive East, Los Angeles, CA,

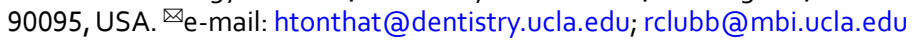


A
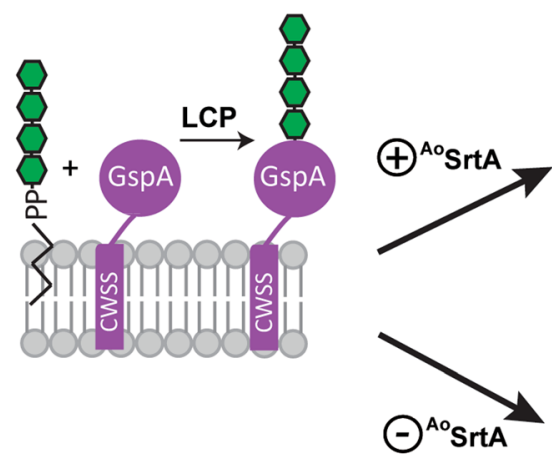
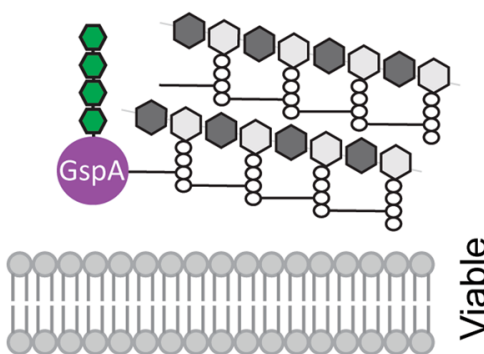

$\frac{\frac{1}{0}}{\frac{0}{5}}$

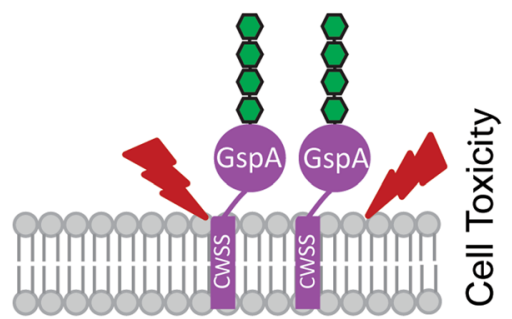

B

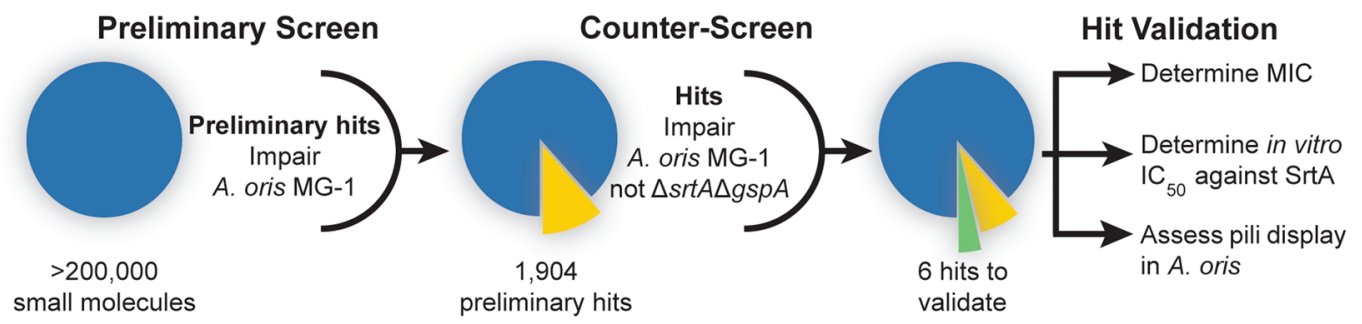

Figure 1. Design and overall work-flow of cell-based inhibitor screen. (A) Schematic showing how the activity of the A. oris SrtA ( ${ }^{\text {Ao } S r t A)}$ enzyme is required for cell viability. A fully functioning AosrtA enzyme is needed to attach the glycosylated GspA protein (colored purple with green glycosylation) to the cell wall (top). Reduced gene expression of the AoSrtA enzyme has been shown to be lethal, presumably because of GspA accumulation in the membrane (bottom). Lethality is dependent upon glycosylation of GspA by the LytR-CpsA-Psr enzymes (LCP). (B) Overview of the sortase inhibitor screen. The effects of small molecules on wild-type A. oris MG-1 was determined for several compound libraries (left). Preliminary hits $(1,904)$ that impaired growth were counter-screened by determining their ability to affect the growth of a $\Delta s r t A / \Delta g s p A$ strain, whose viability is dependent upon the activity of ${ }^{\text {Ao }}$ SrtA (middle). Strain-specific growth inhibitors were then validated for sortase inhibitor activity using biochemical and cellular approaches (right). Adobe Illustrator Version: 15.0.0 (https:// www.adobe.com/products/illustrator.html).

but in some instances these enzymes perform lysine-isopeptide transpeptidation reactions that construct pili virulence factors ${ }^{16}$.

Given its potential as a drug target, because sortase mutants are attenuated in virulence, considerable effort has been put forth to discover small molecule Sa SrtA inhibitors ${ }^{9,17-19}$. Previously reported studies have searched for

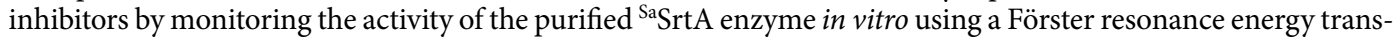
fer (FRET) assay. The FRET assay has been used to screen small-molecule compound libraries ${ }^{20-23}$ and to assess the potency of rationally designed peptidomimetics ${ }^{24-27}$, natural products ${ }^{28-38}$, and small molecules identified using virtual screening approaches ${ }^{39-42}$. While several inhibitors have been discovered, to the best of our knowledge, none have advanced into clinical trials. It is possible that some of these compounds are unable to effectively inhibit the enzyme in its natural context, the extra-cellular bacterial membrane where it may associate with components of the protein secretion and cell wall synthesis machinery. A cell-based assay for high-throughput screening (HTS) for sortase inhibitors could overcome this limitation, but has yet to be implemented in S. aureus because inhibiting SaSrtA activity does not significantly affect the growth or morphology of this microbe in cell culture $^{6}$. SaSrtA activity can be detected in cells, but these methods are cumbersome and require antibody detection of sortase-displayed proteins ${ }^{41}$, cell adhesion assays $s^{43}$ or incubation of cells with fluorogenic peptidyl sortase substrates that can be slow to label cells ${ }^{44}$.

Recently, Wu et al. made the surprising discovery that the viability of the oral bacterium Actinomyces oris MG-1 in cell culture depends on the activity of its sortase ( $\left.{ }^{\text {Ao }} \mathrm{SrtA}\right)^{45,46}$. ${ }^{\text {Ao }} \mathrm{SrtA}$ anchors the glycosylated surface protein A (GspA) to the cell wall. In this process, GspA is first glycosylated by the LCP enzyme and then attached to the cell wall by ${ }^{\text {Ao }}$ SrtA via lipid II (Fig. 1A, top). Interestingly, reducing AoSrtA expression causes cell arrest, presumably due to glycol-stress caused by accumulation of glycosylated GspA in the membrane (Fig. 1A, bottom). To the best of our knowledge, A. oris is the only known bacterium that exhibits a sortase-dependent growth 
phenotype in cell culture. Here we report the development of a cell-based assay to screen for sortase inhibitors that takes advantage of this unique phenotype. High throughput implementation of the assay was used to screen compound libraries and led to the discovery of several small molecule sortase inhibitors that are validated using biochemical and cellular approaches.

\section{Results}

Development and implementation of the cell-based screen. Our previous studies have shown that reducing srtA gene expression in A. oris leads to cell death and that this phenotype is conditionally dependent on $g s p A$ expression ${ }^{45,46}$. To exploit this unique dependence, we developed a cell-based assay to screen for sortase inhibitors that compares the growth-inhibitory effects of small molecules cultured with A. oris MG-1 (wild-type) versus a $\Delta s r t A / \triangle g s p A$ strain. In the assay, it is presumed that a small molecule sortase inhibitor will selectively impair growth of $A$. oris MG-1 by causing GspA to accumulate in the membrane, whereas growth of the $\Delta s r t A$ / $\triangle g s p A$ strain should be unaffected. Prior to implementing the assay in HTS, we optimized conditions for A. oris growth in 384-well plate format. Both MG-1 and $\Delta s t A / \Delta g s p A$ strains had similar growth rates and final cell densities in brain-heart infusion broth. Control growth experiments using media containing $1 \%$ dimethyl sulfoxide (DMSO) confirmed that both strains are tolerant to this solvent at concentrations used for screening. The growth phenotype of both $A$. oris strains was sufficient for use in a HTS, as a Z' score of $0.81(\mathrm{MG}-1)$ and $0.71(\Delta$ srtA/ $\triangle g s p A)$ was determined when positive $\left(1 \mu \mathrm{g} \mathrm{mL}^{-1}\right.$ penicillin) and negative (media only) control experiments are performed $^{47}$.

The overall workflow for the HTS is shown in Fig. 1B. Initially, small molecules were screened for their ability to kill the wild-type MG-1 strain in 384-well format. Molecules that reduced growth by more than 2.75 standard deviations from the average growth were considered preliminary hits. These molecules were then tested in high-throughput for their ability to impair $\Delta s r t A / \triangle g s p A$ growth, which serves as a counter-screen. Small molecules that exhibited differential growth effects in MG-1 but not $\triangle s r t A / \triangle g s p A$ strains were considered hits and carried forward for further analysis. These hits were validated by determining their minimum inhibitory concentrations (MICs) in cell culture, measuring the in vitro half maximal inhibitory concentration of each compound

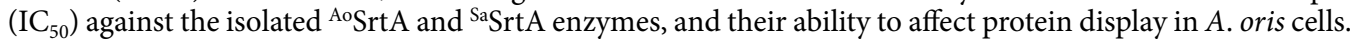

In the primary screen, a total of 200,834 small molecules were tested for their ability to impair A. oris MG-1 growth. Cells were added to an optical density $\left(\mathrm{OD}_{600}\right)$ of 0.01 in media containing $10 \mu \mathrm{M}$ of each molecule, followed by a 15 hour incubation at $37^{\circ} \mathrm{C}$ and end-point $\mathrm{OD}_{600}$ measurement. The growth effect of each molecule was normalized and expressed as a percent growth relative to the DMSO-only control (see Methods). A total of 1,904 molecules were classified as preliminary hits ( $0.95 \%$ of the molecules tested), as they reduced MG- 1 growth after 15 hours by at least $34 \%$, or 2.75 standard deviations below the average. The preliminary hits were then subjected to a counter-screen in which their growth effects on $\Delta s r t A / \Delta g s p A$ were determined. In order to eliminate potentially erroneously identified preliminary hits, the counter-screen and preliminary screen was performed in duplicate for the 1,904 preliminary hit molecules. A molecule was deemed as a hit if it caused greater than $15 \%$ differential growth effect in duplicate when the MG-1 and $\Delta s r t A / \Delta g s p A$ strains were compared. Figure 2 shows a plot of each preliminary hit molecule's growth effect against the MG-1 and $\Delta s r t A / \Delta g s p A$ strains. Data is plotted as the percentage of growth inhibition for each molecule (see Methods).

A total of six compounds preferentially affected MG- 1 growth in duplicate, constituting $0.3 \%$ of the preliminary hits identified from the primary screen and $0.003 \%$ of the total number of compounds tested. The six compounds were termed 1-6 and carried forward for further analysis (Fig. 3). R Version: 3.3.3 (https://www.r-project. $\operatorname{org} /$ )

Evaluation of the sortase inhibitor candidates. Two validation assays were performed for each hit molecule that measured: (i) the minimum inhibitory concentration (MIC) required to prevent growth of the MG-1 and $\Delta s r t A / \Delta g s p A$ strains, and (ii) their in vitro inhibitory activity against the enzymatic activity of the $A$. oris and $S$. aureus sortase enzymes. Initially, the selective growth effect originally observed in the HTS that used 384-well plates was more rigorously defined by determining each molecule's MIC value for the MG-1 and $\Delta s r t A / \Delta g s p A$ strains. Cells were cultured in $100 \mu \mathrm{L}$ media containing 2 -fold dilutions of each small molecule between $320 \mu \mathrm{M}$ and $0.039 \mu \mathrm{M}$ (approximately $134 \mu \mathrm{g} \mathrm{mL}^{-1}$ to $0.16 \mu \mathrm{g} \mathrm{mL}^{-1}$ ). Each of the six preliminary hit molecules (1-6) has a lower MIC for the MG-1 strain (between $16.4 \mu \mathrm{g} \mathrm{mL}^{-1}(40 \mu \mathrm{M})$ and $1.0 \mu \mathrm{g} \mathrm{mL}^{-1}(2.6 \mu \mathrm{M})$ than for $\Delta s r t A / \Delta g s p A$ (Table 1). Two additional molecules exhibited activity in only one replicate of the high-throughput counter-screen assay; however, we chose to interrogate their activity in proceeding assays to better appreciate the reliability of the HTS. Neither exhibited marked differences in their MIC values when tested in MG-1 and $\Delta s r t A / \Delta g s p A$ strains, which affirms the strength of the duplicate experiment cutoff. Thus, the MIC data confirm the results of the HTS by demonstrating that only the duplicate hit molecules preferentially impair the growth of A. oris MG-1.

The ability of the hit molecules to inhibit sortase activity was measured in vitro using an established FRET-based assay ${ }^{13}$ that measures the ability of each enzyme to cleave a fluorogenic peptide substrate; Abz-LAQTG-Dap(Dnp)- $\mathrm{NH}_{2}$ and Abz-LPETG-Dap(Dnp)- $\mathrm{NH}_{2}$, substrates for AoSrtA and Sa SrtA, respectively. Each enzyme cleaves between the threonine and glycine bond in these peptides, leading to a measurable increase in fluorescence (see Methods). The $\mathrm{IC}_{50}$ value of each compound was determined for both the ${ }^{\text {Ao }}$ SrtA and ${ }^{\text {SaSrtA }}$ enzymes. Compounds 3, 4 and $\mathbf{6}$ are bona fide enzyme inhibitors, as each inhibits both the Ao SrtA and ${ }^{\text {SaSrtA }}$ enzymes with $\mathrm{IC}_{50}$ values ranging from $30-70 \mu \mathrm{g} \mathrm{mL}^{-1}(73-170 \mu \mathrm{M})$ (Table 1). In contrast, molecules 1, 2 and 5 failed to inhibit ${ }^{\text {Aos }}$ SrtA in vitro, suggesting that they selectively affect $A$. oris MG-1 growth through a mechanism not involving sortase inhibition.

Sortase inhibitors affect pilus assembly in $\boldsymbol{A}$. oris. The MIC and $\mathrm{IC}_{50}$ data suggest that at least three of the hit molecules are capable of inhibiting AosrtA either within the context of the cell or as an isolated 
A

A. oris growth inhibition

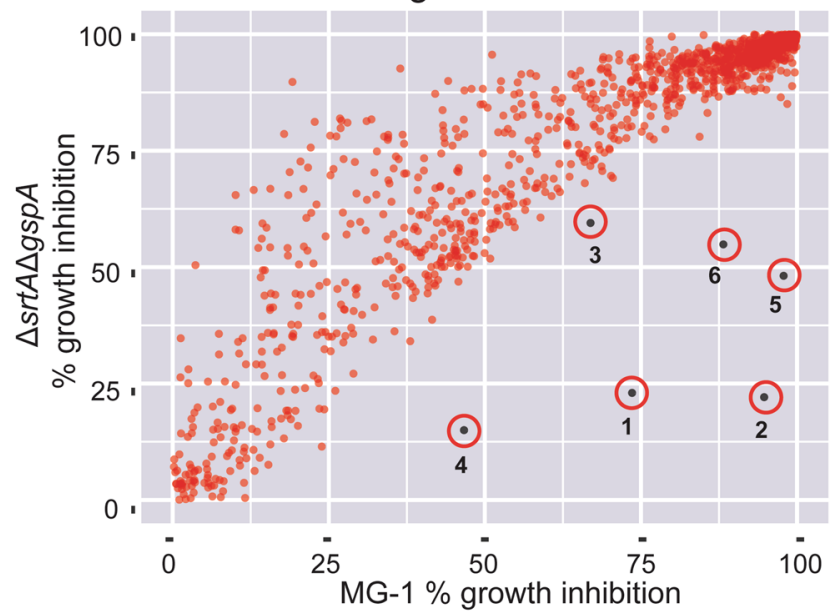

B

Molecule 6 MIC against $A$. oris

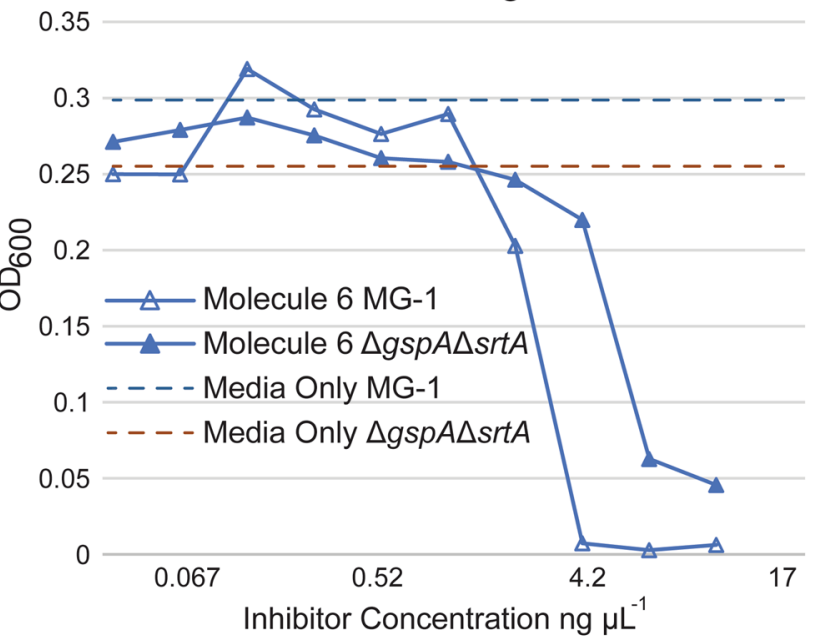

Figure 2. Growth effects of the screened molecules. (A) Scatter plot showing the growth effects of small molecules when cultured with either $A$. oris wild-type MG-1 or mutant strain $\Delta s r t A / \Delta g s p A$. The percent growth inhibition for each strain is relative to the parent strain cultured in the absence of the small molecule (Methods). Molecules (1-6) exhibited statistically significant strain-specific effects on growth, impairing growth of the wild-type more than the $\Delta s t A / \Delta g s p A$ strain (circled and labeled). (B) Plot showing the effects of molecule 6 on the growth $A$. oris MG-1 (open triangles) and $\Delta s r t A / \Delta g s p A$ (filled triangles) strains. The corresponding strains grown in identical conditions in the absence of molecule exhibited $\mathrm{OD}_{600}$ values of 0.29 and 0.26 , respectively. The data show that 6 has a lower MIC for MG-1 as compared to $\Delta s r t A / \Delta g s p A$, and is consistent with the HTS data shown in panel (A).

transpeptidase. To understand the physiological effects that these molecules have on the display of pili and surface proteins on the surface of $A$. oris, we grew cells with $10 \mu \mathrm{M}$ of each hit molecule and assessed sortase activity by western blot and electron microscopy as previously reported ${ }^{45}$. To avoid a confounding problem that inhibiting ${ }^{\text {Ao } S r t A}$ causes cell arrest, we performed the experiments in a mutant devoid of $g s p A$; the aforementioned genetic suppressor of sortase lethality. Consistent with both $\mathrm{MIC}$ and $\mathrm{IC}_{50}$ data, when samples were immunoblotted with antibodies against the type 2 pilus shaft protein FimA, both molecules $\mathbf{3}$ and $\mathbf{4}$ decreased FimA polymers as compared to the control (Fig. 4; compare the first two lanes with lanes containing molecules \#3 and \#4).

To examine if these molecules inhibit cell wall anchoring of surface proteins (e.g. GspA), we similarly treated cells devoid of $l c p A$, i.e. $\Delta l c p A$, another suppressor of $s r t A^{46}$. When blotted with antibodies against GspA, no GspA polymers were observed in samples treated with molecules 3, 4 and 6 (Fig. 4B). To corroborate these observations, we subjected $A$. oris cells of $\Delta g s p A$ and $\Delta l c p$ strains to $10 \mu \mathrm{M}$ of each molecule $(3,4$ and $\mathbf{6})$ and studied pilus display with transmission electron microscopy (TEM), whereby A. oris cells were immobilized on nickel grids and stained with $1 \%$ uranyl acetate prior to viewing by an electron microscope. Intriguingly, compared to the untreated control, treatment of molecules 3,4 and $\mathbf{6}$ caused significant reduction of pilus assembly at the cell 
<smiles>Cc1ccc(S(=O)(=O)Cc2nc3cc(-c4ccccc4)sc3c(=O)[nH]2)cc1</smiles>

4<smiles>COc1ccc2nc(N(Cc3ccccc3)C(=O)c3ccc([N+](=O)[O-])o3)sc2c1</smiles><smiles>Cc1cc(NS(=O)(=O)c2ccc(C(C)(C)C)cc2)n(C2CCS(=O)(=O)C2)n1</smiles>

5<smiles>O=C(c1cc(-c2ccc(F)cc2)n[nH]1)N1CC(c2nc(-c3ccccc3)no2)C1</smiles><smiles>CCc1ccc2nc(N(Cc3cccnc3)C(=O)c3ccc([N+](=O)[O-])o3)sc2c1</smiles>

6<smiles>Cc1nc(COc2ccc(F)cc2)sc1CC(=O)Nc1nc2c(s1)CC(C)CC2</smiles>

Figure 3. Chemical structures of preliminary hit molecules. Chemical structures of the hit molecules (1-6) from the $A$. oris screen. These molecules exhibit statistically significant strain-specific effects on growth, impairing the wild-type more than the $\Delta s r t A / \Delta g s p A$ strain. Compounds 3, 4 and $\mathbf{6}$ were validated sortase inhibitors, whereas 1, 2 and $\mathbf{5}$ selectively impair growth through an unknown, non-sortase dependent mechanism. ChemDraw Professional Version:19.0.1.28 (https://www.perkinelmer.com/product/chemdrawprofessional-chemdrawpro).

\begin{tabular}{|c|c|c|c|c|c|c|}
\hline & \multicolumn{6}{|c|}{ Preliminary Hits } \\
\hline & $\underline{1}$ & $\underline{2}$ & $\underline{3}$ & $\underline{4}$ & $\underline{5}$ & $\underline{6}$ \\
\hline Differential Growth $^{\mathrm{a}}$ & $65.7 \pm 0.5 \%$ & $96.2 \pm 0.3 \%$ & $17.5 \pm 3.5 \%$ & $38.5 \pm 0.5 \%$ & $96.2 \pm 0.3 \%$ & $71.8 \pm 16.5 \%$ \\
\hline $\operatorname{MIC}(\mathrm{MG}-1)^{\mathrm{b}}$ & 2.0 & 0.5 & 8.2 & 16.4 & 1.0 & 2.1 \\
\hline $\operatorname{MIC}(\Delta g s p A / \Delta s r t A)^{\mathrm{b}}$ & 4.0 & 2.1 & $>130$ & $>130$ & 2.0 & 8.4 \\
\hline $\mathrm{IC}_{50}(\text { A. oris })^{\mathrm{c}}$ & $>190$ & $>200$ & $30 \pm 20$ & $70 \pm 50$ & $>190$ & $60 \pm 40$ \\
\hline $\mathrm{IC}_{50}(S . \text { aureus })^{\mathrm{c}}$ & $80 \pm 20$ & $>200$ & $90 \pm 20$ & $200 \pm 100$ & $110 \pm 30$ & $54 \pm 8$ \\
\hline
\end{tabular}

Table 1. Growth and inhibitory properties of the preliminary hit molecules. ${ }^{a}$ Differential effects of small molecule on wild-type MG-1 and $\triangle g s p A \Delta s r t A$ A. oris strains measured in the high-throughput screen. See methods section complete definition. ${ }^{b}$ Minimum inhibitor concentration (MIC) expressed in $\mu \mathrm{g} \mathrm{ml}^{-1}$ for MG-1 and $\Delta g s p A \Delta s r t A$ A. oris strains. ${ }^{~}$ Half maximal inhibitory concentration for the in vitro enzyme activity of ${ }^{\text {Sa SrtA }}$ (S. aureus) or ${ }^{\text {Ao }}$ SrtA (A. oris) in $\mu \mathrm{g} \mathrm{ml}^{-1}$.

pole in either strain background, i.e. $\triangle g s p A$ or $\triangle l c p A$ (Fig. 5). The results suggest that these molecules target the nascent pilus assembly machine.

\section{Discussion}

Sortase enzymes are promising drug targets as they mediate the display of important virulence factors in several medically significant bacteria. The S. aureus Sa SrtA enzyme is particularly interesting because it plays an important role in MRSA infections that are a major cause of human mortality. Despite over fifteen years of effort by a number of research groups, antivirulence agents that work by inhibiting SastA have yet to be discovered. This may be because nearly all screens for SaSrtA inhibitors have used the isolated enzyme that is truncated at its $\mathrm{N}$-terminus to increase solubility. Searching for inhibitors using a cell-based assay may yield better results as it could discover small molecules that are capable of traversing the cell wall and targeting the intact enzyme in the microbial membrane. Moreover, it could overcome screening limitations that are caused by the slow enzyme kinetics of the isolated protein. To the best of our knowledge, a robust cell-based sortase assay has yet to be developed, presumably because current methods used to monitor Sa SrtA activity in intact cells are laborious, requiring multi-step immunolabeling and fluorescence-detection experiments. To overcome this limitation, we developed and implemented in high throughput a cell-based assay to identify sortase inhibitors. Our approach exploits the unique growth dependence of $A$. oris on the activity of its SrtA enzyme, which is conditionally dependent on glycosylation of the GspA surface protein by an LCP enzyme (Fig. 1A). First, a primary screen was performed in which $>200,000$ small molecules were tested for their ability to impair the growth of wild-type A. oris MG-1 (Fig. 1B). Preliminary hit molecules were then counter-screened against the A. oris $\Delta s r t A / \Delta g s p A$ mutant strain to eliminate small molecules that impaired $A$. oris growth via processes unrelated to sortase. In the primary screen, 

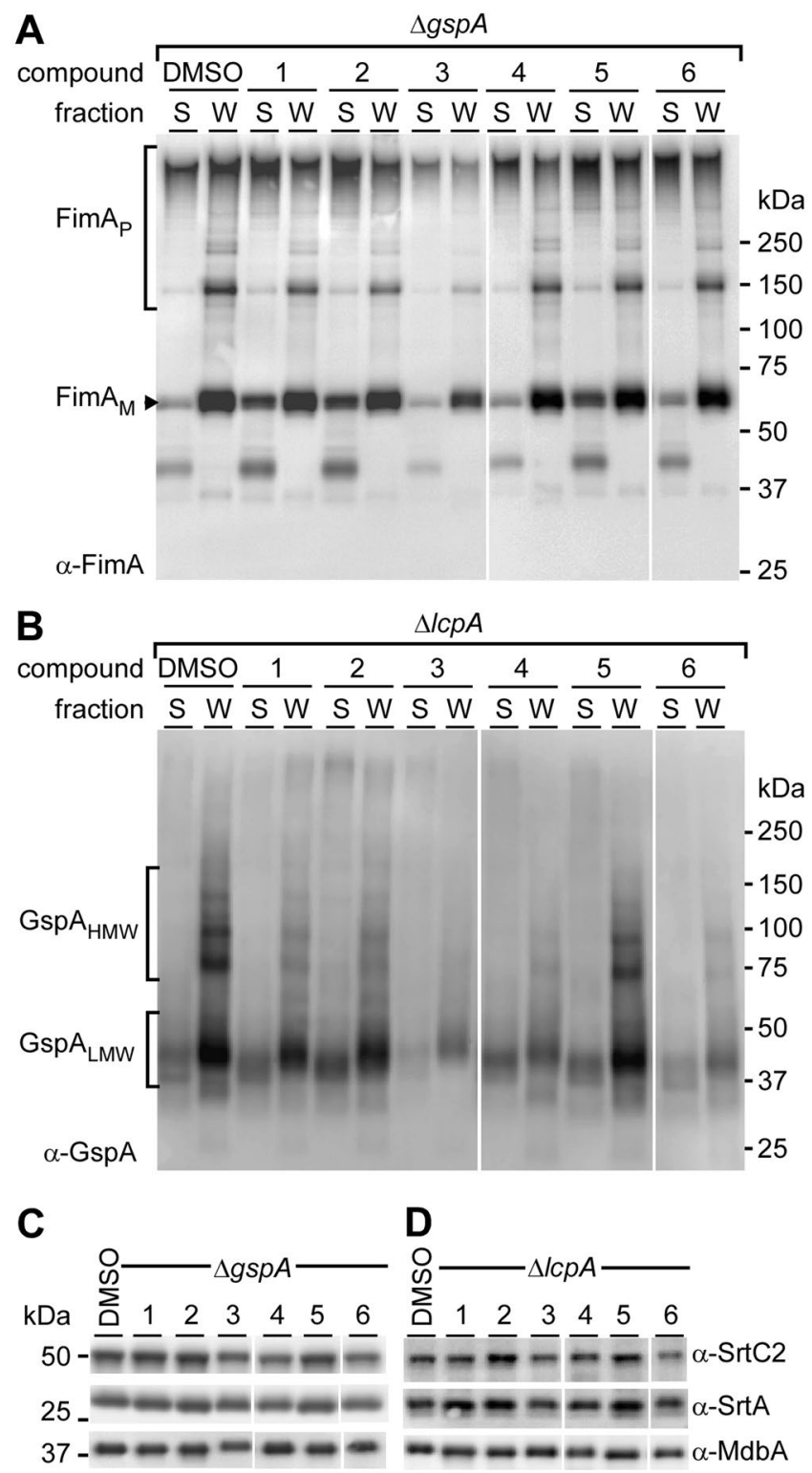

Figure 4. Assessment of cell surface proteins. (A,B) Cell cultures of A. oris strains $\Delta g s p A(\mathbf{A})$ and A. oris $\Delta l c p A$ (B) harvested after inhibitor treatment were subjected to cell fractionation. Samples in supernatant culture medium (S) and cell wall (W) fractions were immunoblotted with antibodies against the fimbrial shaft FimA $(\alpha$-FimA $)$ and glycosylated GspA $(\alpha-G s p A)$. (C,D) The protoplast fractions were subjected to immunoblotting with antibodies against pilus-specific sortase SrtC2 ( $\alpha$-SrtC2), housekeeping sortase SrtA ( $\alpha-S r t A)$, and the membrane protein $\mathrm{MdbA}(\alpha-\mathrm{MdbA})$ as control.

most molecules did not affect $A$. oris MG-1 growth when present in the cell culture at $10 \mu \mathrm{M}$, as the mean and median percent growth of cultures containing small molecules relative cells grown in standard growth culture containing DMSO was $99.3 \%$ and $100.9 \%$, respectively. The interquartile range (IQR) is $12.1 \%$, meaning that $75 \%$ of molecules cells grow to $94.2 \%$ the average DMSO-only density or better. A total of 1,904 molecules in the screen reduced MG-1 growth to $66 \%$ growth (2.75 standard deviations below the average percent growth) and were subsequently counter-screened using the $\Delta s r t A / \Delta$ gspA mutant strain. For these molecules, growth effects on each strain were tested in duplicate on separate days to rigorously identify molecules that selectively impaired MG-1 growth. Six preliminary hit molecules were identified, 1-6 (0.32\% of the 1,904 MG-1 growth effecting molecules). Each of the six molecules inhibit the growth of the MG-1 strain more than the $\Delta g s p A / \Delta s r t A$ strain when tested in duplicate. The finding that few molecules cause selective growth effects is not surprising, as most molecules can be expected to be generally cytotoxic by inhibiting other essential non-sortase pathways within cell, e.g. division and respiration.

Repeating the counter-screen in duplicate significantly eliminated false positive results. This is evident by our finding that the six preliminary hits also exhibit strain-specific growth effects when they were re-purchased and tested in larger culture volumes (Table 1); each of the preliminary hits (1-6) exhibited lower MIC values against 


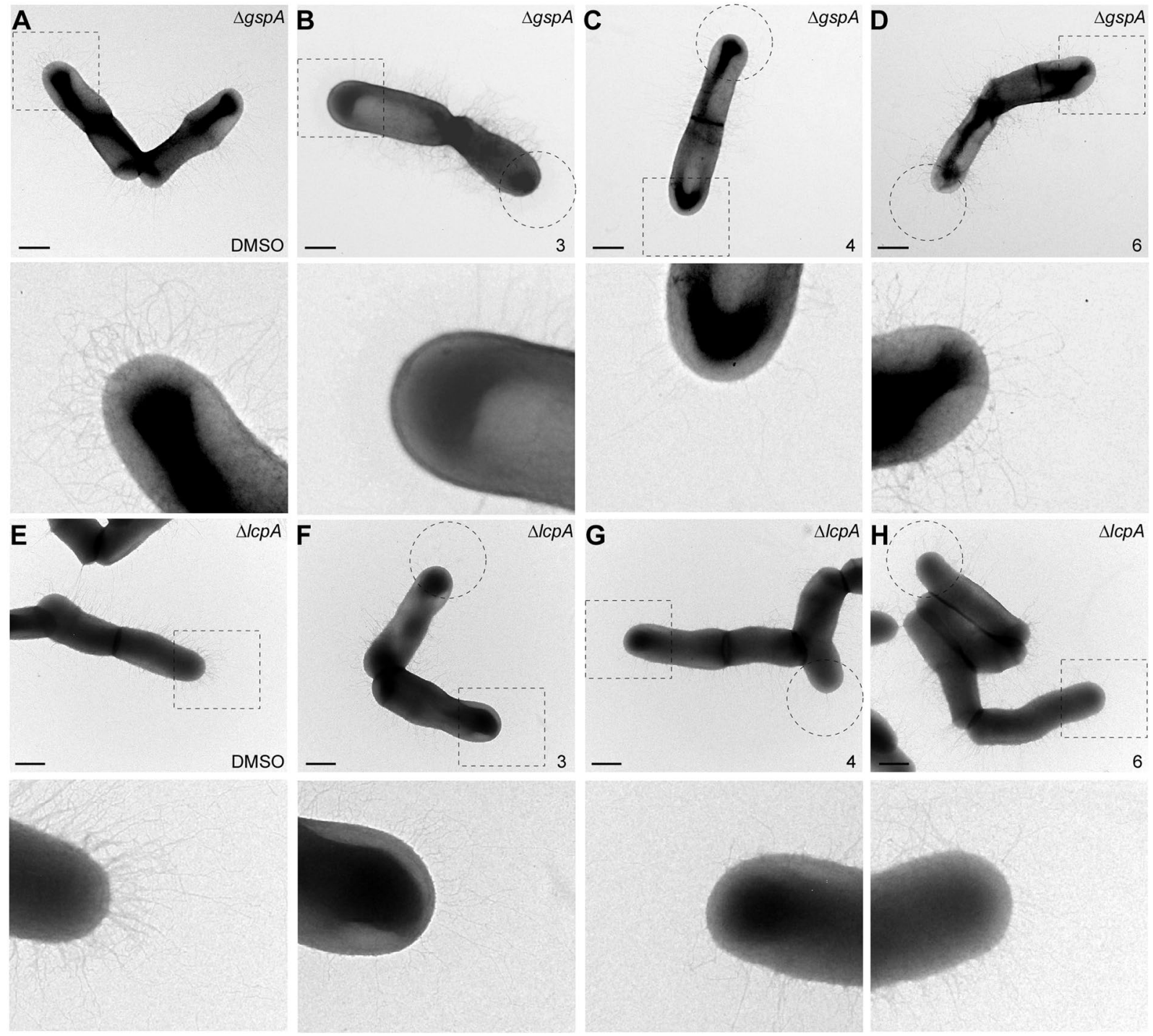

Figure 5. Detection of pili by electron microscopy. Bacterial cultures of strains $\Delta g s p A(\mathbf{A}-\mathbf{D})$ and $\Delta l c p A(\mathbf{E}-\mathbf{H})$ treated with compounds 3, 4 and $\mathbf{6}$ were subjected to negative staining with $1 \%$ uranyl acetate prior to electron microscopy; scale bar of $500 \mathrm{~nm}$. Polar assembly of pili is heighted with dashed circles and rectangles. Enlarged areas in dashed rectangles are shown in below panels.

MG-1 than against $\Delta g s p A / \Delta s r t A$. The MIC values against MG-1 fall within a similar range, around $10 \mu \mathrm{M}$ for all hits, which is expected, as the primary screen and counter-screen monitored growth effects at a single concentration. It is worth noting that the growth screen may have failed to detect potential sortase inhibitors with MIC values significantly greater than $10 \mu \mathrm{M}$, meaning higher concentrations of the small molecule are necessary for enzyme inhibition. In principle, this limitation could be overcome by repeating the HTS using higher concentrations of the small molecules in the screen, but this was not performed here because it was cost-prohibitive.

In vitro enzyme activity testing reveals that compounds 3, 4 and 6 inhibit both the Ao SrtA and ${ }^{\text {Sa SrtA enzymes }}$ with $\mathrm{IC}_{50}$ values ranging from 30 to $70 \mu \mathrm{g} \mathrm{mL}^{-1}(73-170 \mu \mathrm{M})$. Compounds 3 and 4 are bioisosteres and share related $\mathrm{N}$-(1,3-benzothiazol-2-yl) -5-nitrofuran-2-carboxamide chemical scaffold. In compound 3 the scaffold is elaborated with N-3-pyridylmethyl and 6-ethyl-2-benzothiazole moieties, whereas 4 features benzyl and 5 -methoxybenzothiazole. Effectively these compounds are bioisosteres, as the benzene is an isostere with pyridine, as are the ethyl and methoxy groups. Interestingly, molecules containing 5-nitrofuran functional groups are particularly potent at eliciting selective growth effects as they are enriched in our primary hits; $1 \%$ of the 1,904 hits from the cell based screen contained this functional group even though it is present in only $0.09 \%$ of 200,834 molecules that we screened. Compound 6 is unique, exhibiting a N-methyl-(4,5,6,7-tetrahydro-1,3-benzothiazol2-yl)-1,3-thiazole-5-carboxamide scaffold. Importantly, compound 6 would not likely have been discovered using conventional FRET-based HTS as it is intrinsically fluorescent and presumably would have been disregarded as a hit. The $\mathrm{IC}_{50}$ values for the inhibitors against ${ }^{\text {Ao }}$ SrtA are higher than their measured MIC values. This suggests that they may also kill $A$. oris cells through non-sortase related mechanisms and/or that they more efficiently inhibit the A. oris enzyme when it is embedded in the microbial membrane. Preliminary hits $\mathbf{1}, \mathbf{2}$ and $\mathbf{5}$ also effect growth 
in a strain-specific manner, but do not inhibit ${ }^{\text {Ao } S r t A ~ i n ~ v i t r o ~(T a b l e ~ 1) . ~ T h e ~ o r i g i n s ~ o f ~ t h e i r ~ s e l e c t i v e ~ e f f e c t s ~ o n ~}$ growth remain to be determined. To the best of our knowledge, none of the hit molecules have been described as antimicrobials, however benzothiazole-based DNA gyrase B inhibitors have been shown to have modest growth inhibitor properties against Gram-positive Entereoccocus faecalis when dosed at high concentrations $(50 \mu \mathrm{M})^{48}$.

Cellular studies indicate that sortase inhibitors 3, 4 and $\mathbf{6}$ alter the ability of $A$. oris to display GpsA and to assemble pili on its surface. A. oris uses three distinct sortases to elaborate its cell envelope. Ao SrtA is a class $\mathrm{E}$ housekeeping sortase that attaches GspA and other proteins containing the LAQTG sorting signal to the cell wall $^{49}$. Ao SrtA catalyzes a transpeptidation reaction that attaches GspA to lipid II, a cell wall precursor. A. oris also assembles surface pili (fimbriae) using two class C sortases, SrtC1 and SrtC2. These sortases catalyze transpeptidation by linking protein subunits of the pilus together via lysine-isopepide bonds. Subsequent cell wall anchoring of pilus polymers to peptidoglycan requires ${ }^{\text {Ao }} \mathrm{SrtA}^{49}$. SrtC1 produces type 1 fimbriae that are comprised of the fimbrial shaft FimP protein and the tip fimbrillin FimQ protein. This structure mediates bacterial adherence to the tooth surface via FimQ interactions with salivary proline-rich protein deposits. The SrtC2 sortase produces type 2 fimbriae, made of the fimbrial shaft FimA protein and tip fimbrillin FimB; these fimbriae are required for bacterial adherence to host cells, biofilm formation, and bacterial coaggregation ${ }^{50,51}$. We performed cell fraction and immunoblot studies to gain insight into how inhibitors 3, 4 and $\mathbf{6}$ affect SrtC1 and Ao SrtA sortase activity in intact cells (Fig. 4). Molecule 6 inhibits ${ }^{\text {Ao }}$ SrtA in cells as its presence significantly diminishes GspA display (Fig. 4B). In contrast, it has little effect on type 2 pilus production by the SrtC2 sortase as judged by immunoblots of the FimA shaft protein in fractionated cells. The diminished potency of molecule 6 against pili display indicates that it lacks inhibitory activity against class $\mathrm{C}$ sortases. This is substantiated by TEM images, which show that molecule 6 only modestly effects pili display in the $\Delta g s p A$ strain (Fig. 5A-D). Since ${ }^{\text {Ao }}$ SrtA has been implicated in anchoring of the pilus to the peptidoglycan, one might still expect to see diminished pili display in the absence of the housekeeping sortase; however, it is not uncommon for class C enzymes to compensate for loss of the housekeeping sortase and independently display pili as SrtC2 is capable of catalyzing pilus polymerization and cell wall anchoring of pilus polymers ${ }^{49,52}$. Thus, the data supports molecule 6 as being more selective for ${ }^{\text {Ao }}$ SrtA in intact cells.

Cellular studies suggest that compounds 3 and 4 inhibit both class E AoSrtA and class C SrtC2 sortases. Our interrogation of type 2 pilus assembly reveals that 3 and $\mathbf{4}$ not only decrease the abundance of pili, but also reduce the amount of higher molecular weight FimA-containing polymers that are being formed (Fig. 4A, compare molecular weight bands $\geq 150 \mathrm{kDa}$ ). Decreased pilin-polymerase activity suggests that SrtC2 is being inhibited. This is substantiated by TEM images of cells treated with these compounds $\mathbf{3}$ and $\mathbf{4}$, which show either completely absent or diminished pili at their poles, respectively (Fig. 5B,C). Inspection reveals that the pili in these cells become gradually shorter toward the poles, suggesting that the SrtC2 enzyme is being inhibited during the 3 hour period of growth. Moreover, cells treated with compounds $\mathbf{3}$ and $\mathbf{4}$ have decreased amounts of cell wall associated GspA, compatible with the fact that these molecules also inhibiting the housekeeping AoSrtA sortase (Fig. 4B). Consistent with the lower $\mathrm{IC}_{50}$ against ${ }^{\mathrm{Ao}} \mathrm{SrtA}$ and ${ }^{\mathrm{Sa}} \mathrm{SrtA}$ and the lower MIC against MG-1 relative to 4, we note that 3 appears to more severely limit GspA display than 4 at $10 \mu \mathrm{M}$ (Figs. $4 \mathrm{~B}$ and 5B,C). This suggests that 3, an analog of 4 , is the more potent molecule. It is important to note that these inhibitors were added to actively growing cell cultures that already produced pili. Given the presence of pili at the septal area and significantly reduced pili at the pole (Fig. 5), we surmise that these inhibitors target the new sortase machine at the pole, where the nascent peptidoglycan is synthesized and modified.

In conclusion, we have exploited the unique sortase-dependent growth phenotype of A. oris to screen small molecule compound libraries for sortase inhibitors. Three molecules, representing two unique scaffolds, inhibit sortase enzymes from both A. oris and S. aureus were discovered. This represents the first HTS for sortase inhibitors that relies on the simple metric of cellular growth and suggests that $A$. oris is a promising platform for sortase-targeted drug discovery. Future work will need to establish structure-activity relationships for the hit molecules to further increase their potency for potential use as novel anti-infectives, which are urgently needed to treat infections caused by MRSA and other drug-resistant bacterial pathogens.

\section{Materials and Methods}

Antibiotics, media and consumables. Brain Heart Infusion Broth, modified (BHI) was purchased from Fisher Scientific and prepared as directed within one week of its use. Penicillin G and kanamycin were purchased from Fisher Scientific and stored as directed. 1000x antibiotic stocks were prepared in water, filtered with $0.2 \mu \mathrm{m}$ syringe filters, and stored at $-20^{\circ} \mathrm{C}$ until thawed immediately before use. Greiner 384-well plates (EK-30162) and universal lids (EK-2079) were purchased from E\&K scientific and used once before disposal as medical waste.

High-throughput screen. A total of 200,834 small molecule compounds (Molecular Screening Shared Resource, UCLA) were dissolved in 100\% Omnisolv methylsulfoxide (MilliporeSigma MX1456P-6) and stored in 384-well polypropylene plates. Actinomyces oris MG-1 and $\Delta g s p A \Delta s r t A$ were streaked out on BHI agar plates containing $50 \mu \mathrm{g} / \mathrm{mL}$ kanamycin and grown at $37^{\circ} \mathrm{C}$ for 48 hours to allow single colonies to grow. Cultures were started from 2-3 colonies in BHI with $50 \mu \mathrm{g} / \mathrm{mL}$ kanamycin and allowed to grow until an $\mathrm{OD}_{600}$ between $0.1-0.4$. Meanwhile, 384-well plates were filled with $25 \mu \mathrm{L}$ BHI broth with $100 \mu \mathrm{g} \mathrm{mL}{ }^{-1}$ kanamycin (columns $1-22$ ) or $200 \mu \mathrm{g} \mathrm{mL}^{-1}$ Penicillin G (positive control, columns 23-24). Five hundred nanoliters of small molecules were transferred into the media (columns 3-22) using the Biomex $\mathrm{FX}^{\mathrm{P}}$ automated work station with a 384-well pin tool. Precultures were diluted to an $\mathrm{OD}_{600}$ of 0.02 in $1 \mathrm{~L} \mathrm{BHI}$ broth without antibiotics. Twenty five microliters of cell culture was immediately aliquoted into plates containing $25 \mu \mathrm{L}$ media, antibiotics, and small-molecules using a Multidrop (Thermo LabSystems), resulting in $50 \mu \mathrm{g} \mathrm{mL}^{-1}$ kanamycin and $10 \mu \mathrm{M}$ small molecules with $1 \%$ DMSO or $100 \mu \mathrm{g} \mathrm{mL}^{-1}$ Penicillin G. Plates were immediately lidded and placed into a humidified Cytomat 6001 incubator at $37^{\circ} \mathrm{C}$ and allowed to grow for 15.5 hours. Following incubation, plates were removed from the 
Cytomat using a Thermo Spinnaker robotic arm on a rail, de-lidded, and placed into an EnVision high-speed plate reader for optical density measurement at $620 \mathrm{~nm}$.

Raw data obtained from the screen was formatted in-house to upload to the Collaborative Drug Discovery Vault (www.collaborativedrug.com). Individual optical density readings were converted to a percent growth value $\left(\left[\mathrm{OD}_{\text {sample }}-\mathrm{OD}_{\text {Positive control }}\right] /\left[\mathrm{OD}_{\text {Negative Control Average }}-\mathrm{OD}_{\text {Positive control }}\right]\right)$ and percent growth inhibition (1-percent growth). The Z-factors for the individual molecules were determined. Molecules with a Z-factor less than or equal to -2.75 were considered preliminary hits to advance forward a generous collection of preliminary hit molecules. Five microliters of each of the 1904 preliminary hit molecules were re-arrayed into new 384-well polypropylene plates. Each of the 1904 preliminary hits were assayed against both the MG-1 and $\Delta g s p A \Delta s r t A$ strains in duplicate, on two separate days. Percent difference of growth was calculated according to the equation 100$\left.100\left[\left(\mathrm{OD}_{\mathrm{MG}-1, \text { Sample }}-\mathrm{OD}_{\mathrm{MG}-1 \text {, Positive Control }}\right) / \mathrm{OD}_{\Delta g s p A \Delta s r t A \text {, Sample }}-\mathrm{OD}_{\Delta g s p A \Delta s r t A \text {, Positive Control }}\right)\right] /\left[\left(\mathrm{OD}_{\mathrm{MG}-1, \text { Average }}-\mathrm{OD}_{\mathrm{MG}-1 \text {, }}\right.\right.$ Positive Control $\left.\left.) / \mathrm{OD}_{\triangle g s p A \Delta s t t A, \text { Average }}-\mathrm{OD}_{\triangle g s p A \Delta s r t A, \text { Positive Control }}\right)\right]$. A score of 100 indicates that a molecule causes extreme difference in cell survivability between the two strains whereas a score of 0 indicates no differential effect on cellular growth. Molecules were ranked according to their relative growth.

Hit validation assays. Minimum inhibitory concentration (MIC) values were determined for molecules 1-6 according to the Clinical Laboratory Standard Institutes' (CLSI) Methods for Dilution Antimicrobial Susceptibility Tests for Bacteria that Grow Aerobically, Approved standards-ninth edition; M07-A9 Vol. 32 No. 2 with the following alterations. Overnight cultures of MG-1 and $\Delta g s p A \Delta s r t A$ were diluted to $\mathrm{OD}_{600}$ values of 0.01 before being added to BHI containing the appropriate molecule in 96-well plates. Mueller-Hinton broth was not usable because $A$. oris is a fastidious bacterium. Plates were sealed with Breatheasy seals and incubated for 18 hours, as $A$. oris has a doubling time of 2 hours. Plate seals were removed and $\mathrm{OD}_{600}$ values measured. Each plate was run with a positive control (cells, media, and Penicillin G), negative control (media and cells only), and contamination control (media only).

$\mathrm{IC}_{50}$ values for the six hit molecules against the AosrtA and Sa SrtA enzymes were determined as previously described, with some modifications. Briefly, molecules 1-6 were serial diluted 2-fold (from 1.25 mM to 2.44 $\mu \mathrm{M}$, $500 \mu \mathrm{M}$ to $0.98 \mu \mathrm{M}$ final assay concentration) into $25 \mu \mathrm{M}{ }^{\text {Ao } S r t A ~ o r ~ S a S r t A ~(f i n a l ~ a s s a y ~ c o n c e n t r a t i o n ~} 10 \mu \mathrm{M}$ ) in buffer A (20 mM HEPES pH 7, $5 \mathrm{mM} \mathrm{CaCl}_{2}, 0.05 \%$ TWEEN, 30\% DMSO). The final DMSO concentration was $18 \%$ in the assay. Samples were incubated 1 hour at room temperature and aliquoted into a 384-well plate (EK-

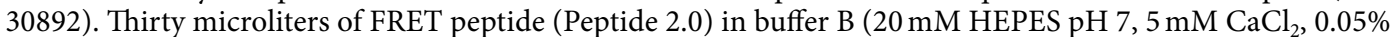
TWEEN), Abz-LPATG-Dap(Dnp)- $\mathrm{NH}_{2}$ for ${ }^{\mathrm{Sa}}$ SrtA or Abz-LAQTG-Dap(Dnp)- $\mathrm{NH}_{2}$ for ${ }^{\text {Aos }}$ SrtA was added and the plate was immediately placed into a Flexstation II plate reader (Molecular Devices) and fluorescence was recorded at $335 / 420 \mathrm{~nm}$ excitation/emission wavelengths after 5 seconds of plate shaking to mix the reaction components. Plate fluorescence was measured over the course of 30 minutes.

Cell fractionation and western blotting. Cell fractionation and Western blotting were followed according to a published protocol ${ }^{53}$. Briefly, the cultures of $A$. oris strains grown in $\mathrm{BHI}$ at $37^{\circ} \mathrm{C}$ until $\mathrm{OD}_{600}$ of 0.25 were aliquoted, and bacterial aliquots were treated with $10 \mu \mathrm{M}$ of individual sortase inhibitors for 3 hours. Bacterial cultures were then normalized to equal $\mathrm{OD}_{600}$, and cells were fractionated into culture medium (S), cell wall (W), and protoplast fractions. Isolated fractions were subjected to protein precipitation by $7.5 \%$ trichloroacetic acid, followed by washing with cold acetone, except for the protoplast fractions. Protein samples were dissolved in hot sodium dodecyl sulfate (SDS)-containing sample buffer, separated by 3-12\% Tris-glycine gradient gels, and subjected to immunoblotting with specific antisera ( $\alpha$-FimA, 1:10,000 dilution; $\alpha-G s p A, \alpha-M d b A, \alpha-S r t C 2$ and $\alpha$-SrtA, 1:4000 dilution), followed by chemo-luminescence detection.

Transmission electron microscopy. To observe cell morphology by negative staining, A. oris cells harvested after inhibitor treatment were washed once with PBS and suspended in $0.1 \mathrm{M} \mathrm{NaCl}$. A drop of $7 \mu \mathrm{L}$ of bacterial suspension was placed onto carbon-coated nickel grids and stained with $1 \%$ uranyl acetate. Samples were examined using a JEOL JEM1200.

\section{Data availability}

All data from this study are available from the corresponding author.

Received: 23 December 2019; Accepted: 20 April 2020;

Published online: 22 May 2020

\section{References}

1. Fischetti, V. A. Surface Proteins on Gram-Positive Bacteria. Microbiol Spectr 7(4), GPP3-0012 (2019).

2. Navarre, W. W. \& Schneewind, O. Surface proteins of gram-positive bacteria and mechanisms of their targeting to the cell wall envelope. Microbiol Mol Biol Rev 63(1), 174-229 (1999).

3. Siegel, S. D., Reardon, M. E. \& Ton-That, H. Anchoring of LPXTG-Like Proteins to the Gram-Positive Cell Wall Envelope. Curr Top Microbiol Immunol 404, 159-175 (2017)

4. Jacobitz, A. W., Kattke, M. D., Wereszczynski, J. \& Clubb, R. T. Sortase Transpeptidases: Structural Biology and Catalytic Mechanism. Adv Protein Chem Struct Biol 109, 223-264 (2017).

5. Spirig, T., Weiner, E. M. \& Clubb, R. T. Sortase enzymes in Gram-positive bacteria. Mol Microbiol 82(5), 1044-1059 (2011).

6. Mazmanian, S. K., Liu, G., Ton-That, H. \& Schneewind, O. Staphylococcus aureus sortase, an enzyme that anchors surface proteins to the cell wall. Science 285(5428), 760-763 (1999).

7. Lee, A. S. et al. Methicillin-resistant Staphylococcus aureus. Nat Rev Dis Primers 4, 18033 (2018).

8. Klein, E. Y. et al. National Costs Associated With Methicillin-Susceptible and Methicillin-Resistant Staphylococcus aureus Hospitalizations in the United States, 2010-2014. Clin Infect Dis 68(1), 22-28 (2019).

9. Maresso, A. W. \& Schneewind, O. Sortase as a target of anti-infective therapy. Pharmacol Rev 60(1), 128-141 (2008). 
10. Frankel, B. A., Kruger, R. G., Robinson, D. E., Kelleher, N. L. \& McCafferty, D. G. Staphylococcus aureus sortase transpeptidase SrtA: insight into the kinetic mechanism and evidence for a reverse protonation catalytic mechanism. Biochemistry 44(33), 11188-11200 (2005).

11. Ilangovan, U., Ton-That, H., Iwahara, J., Schneewind, O. \& Clubb, R. T. Structure of sortase, the transpeptidase that anchors proteins to the cell wall of Staphylococcus aureus. Proc Natl Acad Sci U S A 98(11), 6056-6061 (2001).

12. Huang, X. et al. Kinetic mechanism of Staphylococcus aureus sortase SrtA. Biochemistry 42(38), 11307-11315 (2003).

13. Ton-That, H., Mazmanian, S. K., Faull, K. F. \& Schneewind, O. Anchoring of surface proteins to the cell wall of Staphylococcus aureus. Sortase catalyzed in vitro transpeptidation reaction using LPXTG peptide and NH(2)-Gly(3) substrates. J Biol Chem 275(13), 9876-9881 (2000).

14. Comfort, D. \& Clubb, R. T. A comparative genome analysis identifies distinct sorting pathways in gram-positive bacteria. Infect Immun 72(5), 2710-2722 (2004).

15. Dramsi, S., Trieu-Cuot, P. \& Bierne, H. Sorting sortases: a nomenclature proposal for the various sortases of Gram-positive bacteria. Res Microbiol 156(3), 289-297 (2005).

16. Ton-That, H. \& Schneewind, O. Assembly of pili in Gram-positive bacteria. Trends Microbiol 12(5), 228-234 (2004).

17. Cascioferro, S. et al. Sortase A Inhibitors: Recent Advances and Future Perspectives. J Med Chem 58(23), 9108-9123 (2015).

18. Suree, N., Jung, M. E. \& Clubb, R. T. Recent advances towards new anti-infective agents that inhibit cell surface protein anchoring in Staphylococcus aureus and other gram-positive pathogens. Mini Rev Med Chem 7(10), 991-1000 (2007).

19. Cascioferro, S., Totsika, M. \& Schillaci, D. Sortase A: An ideal target for anti-virulence drug development. Microb Pathog 77C, 105-112 (2014).

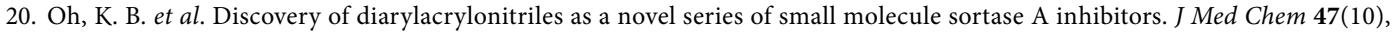
2418-2421 (2004).

21. Suree, N. et al. Discovery and structure-activity relationship analysis of Staphylococcus aureus sortase A inhibitors. Bioorg Med Chem 17(20), 7174-7185 (2009).

22. Jaudzems, K. et al. Targeting Bacterial Sortase A with Covalent Inhibitors: 27 New Starting Points for Structure-Based Hit-to-Lead Optimization. ACS Infect Dis 6(2), 186-194 (2020).

23. Wehrli, P. M. et al. Discovery and development of substituted thiadiazoles as inhibitors of Staphylococcus aureus Sortase A. Bioorg Med Chem 27(19), 115043 (2019).

24. Kruger, R. G., Barkallah, S., Frankel, B. A. \& McCafferty, D. G. Inhibition of the Staphylococcus aureus sortase transpeptidase SrtA by phosphinic peptidomimetics. Bioorg Med Chem 12(13), 3723-3729 (2004).

25. Jung, M. E. et al. Synthesis of (2R,3S) 3-amino-4-mercapto-2-butanol, a threonine analogue for covalent inhibition of sortases. Bioorg Med Chem Lett 15(22), 5076-5079 (2005).

26. Rentero Rebollo, I. et al. Development of Potent and Selective S. aureus Sortase A Inhibitors Based on Peptide Macrocycles. ACS Med Chem Lett 7(6), 606-611 (2016).

27. Wang, J. et al. Oligopeptide Targeting Sortase A as Potential Anti-infective Therapy for Staphylococcus aureus. Front Microbiol 9, 245 (2018).

28. Oh, I. et al. In vitro sortase A inhibitory and antimicrobial activity of flavonoids isolated from the roots of Sophora flavescens. Arch. Pharm Res 34(2), 217-222 (2011).

29. Won, T. H. et al. Brominated aromatic furanones and related esters from the ascidian Synoicum sp. J Nat Prod 75(12), 2055-2061 (2012).

30. Won, T. H. et al. Beta-carboline alkaloids derived from the ascidian Synoicum sp. Bioorg Med Chem 20(13), 4082-4087 (2012).

31. Jeon, J. E. et al. Discorhabdins from the Korean marine sponge Sceptrella sp. J Nat Prod 73(2), 258-262 (2010).

32. Kim, S. H. et al. Inhibition of sortase, a bacterial surface protein anchoring transpeptidase, by beta-sitosterol-3-O-glucopyranoside from Fritillaria verticillata. Biosci Biotechnol Biochem 67(11), 2477-2479 (2003).

33. Kim, S. W., Chang, I. M. \& Oh, K. B. Inhibition of the bacterial surface protein anchoring transpeptidase sortase by medicinal plants. Biosci Biotechnol Biochem 66(12), 2751-2754 (2002).

34. Jang, K. H. et al. Aaptamines as sortase A inhibitors from the tropical sponge Aaptos aaptos. Bioorg Med Chem Lett 17(19), 5366-5369 (2007).

35. Bae, J. et al. Sesterterpenes from the tropical sponge Coscinoderma sp. J Nat Prod 74(8), 1805-1811 (2011).

36. Park, B. S. et al. Curcuma longa L. constituents inhibit sortase A and Staphylococcus aureus cell adhesion to fibronectin. J Agric Food Chem 53(23), 9005-9009 (2005).

37. Younis, S., Taj, S. \& Rashid, S. Structural studies of Staphylococcus aureus Sortase inhibiton via Conus venom peptides. Arch Biochem Biophys 671, 87-102 (2019).

38. Nitulescu, G. et al. Discovery of natural naphthoquinones as sortase A inhibitors and potential anti-infective solutions against Staphylococcus aureus. Drug Dev Res 80(8), 1136-1145 (2019).

39. Chan, A. H. et al. Discovery of Staphylococcus aureus Sortase A Inhibitors Using Virtual Screening and the Relaxed Complex Scheme. Chem Biol Drug Des 82(4), 418-428 (2013).

40. Chenna, B. C. et al. Identification of novel inhibitors of bacterial surface enzyme Staphylococcus aureus Sortase A. Bioorg Med Chem Lett 18(1), 380-385 (2008).

41. Zhang, J.; et al, Antiinfective therapy with a small molecule inhibitor of Staphylococcus aureus sortase. Proc Natl Acad Sci U S A 111 (37), 13517-13522.

42. Nitulescu, G. et al. Molecular Docking and Screening Studies of New Natural Sortase A Inhibitors. Int J Mol Sci 18(10), 2217 (2017).

43. Elgalai, I. \& Foster, H. A. Comparison of adhesion of wound isolates of Staphylococcus aureus to immobilized proteins. J Appl Microbiol 94(3), 413-420 (2003).

44. Nelson, J. W. et al. A biosynthetic strategy for re-engineering the Staphylococcus aureus cell wall with non-native small molecules. ACS Chem Biol 5(12), 1147-1155 (2010).

45. Siegel, S. D. et al. Structure and Mechanism of LcpA, a Phosphotransferase That Mediates Glycosylation of a Gram-Positive Bacterial Cell Wall-Anchored Protein. MBio 10(1), E01580-18 (2019).

46. Wu, C. et al. Lethality of sortase depletion in Actinomyces oris caused by excessive membrane accumulation of a surface glycoprotein. Mol Microbiol 94(6), 1227-1241 (2014).

47. Zhang, J. H., Chung, T. D. \& Oldenburg, K. R. A Simple Statistical Parameter for Use in Evaluation and Validation of High Throughput Screening Assays. J Biomol Screen 4(2), 67-73 (1999).

48. Gjorgjieva, M. et al. Discovery of Benzothiazole Scaffold-Based DNA Gyrase B Inhibitors. J Med Chem 59(19), 8941-8954 (2016).

49. Chang, C. et al. Cell-to-cell interaction requires optimal positioning of a pilus tip adhesin modulated by gram-positive transpeptidase enzymes. Proc Natl Acad Sci U S A 116(36), 18041-18049 (2019).

50. Mishra, A. et al. Two autonomous structural modules in the fimbrial shaft adhesin FimA mediate Actinomyces interactions with streptococci and host cells during oral biofilm development. Mol Microbiol 81(5), 1205-1220 (2011).

51. Mishra, A. et al. The Actinomyces oris type 2 fimbrial shaft FimA mediates co-aggregation with oral streptococci, adherence to red blood cells and biofilm development. Mol Microbiol 77(4), 841-854 (2010).

52. Chang, C. et al. In vitro reconstitution of sortase-catalyzed pilus polymerization reveals structural elements involved in pilin crosslinking. Proc Natl Acad Sci U S A 115(24), E5477-E5486 (2018).

53. Wu, C. et al. Structural determinants of Actinomyces sortase SrtC2 required for membrane localization and assembly of type 2 fimbriae for interbacterial coaggregation and oral biofilm formation. J Bacteriol 194(10), 2531-2539 (2012). 


\section{Acknowledgements}

We thank our laboratory members for critical review of the manuscript and discussions. This work was supported by grants from the National Institutes of Health/National Institute of Allergy and Infectious Diseases Grants R01 AI121360 and AI052217 (R.T.C) and National Institute of Dental \& Craniofacial Research grant DE017382 (H.T-T.). C.K.S. was supported by a Cellular and Molecular Biology Training Grant (Ruth L. Kirschstein National Research Service Award GM007185). S.D.S was supported by NIDCR under Award F31-DE027295 and the Kopchick fellowship from MD Anderson University of Texas Health, Graduate School of Biomedical Sciences.

\section{Author contributions}

J.E.G., M.D.K., H.T.-T. and R.T.C. conceptualized the study. M.D.K. did the preliminary work. J.E.G. and C.K.S. conducted the screen and analysis and followed up with the hit validation assay. C.Y.C., C.G.W. and S.D.S. did the western blotting and cell fractionation studies. S.W.Y. and M.E.J. helped to evaluate each of the hits. R.D. provided the facility for the screen and guidance for implementation. J.E.G., H.T-T. and R.T.C. jointly wrote the article.

\section{Competing interests}

The authors declare no competing interests.

\section{Additional information}

Supplementary information is available for this paper at https://doi.org/10.1038/s41598-020-65256-x.

Correspondence and requests for materials should be addressed to H.T.-T. or R.T.C.

Reprints and permissions information is available at www.nature.com/reprints.

Publisher's note Springer Nature remains neutral with regard to jurisdictional claims in published maps and institutional affiliations.

Open Access This article is licensed under a Creative Commons Attribution 4.0 International License, which permits use, sharing, adaptation, distribution and reproduction in any medium or format, as long as you give appropriate credit to the original author(s) and the source, provide a link to the Creative Commons license, and indicate if changes were made. The images or other third party material in this article are included in the article's Creative Commons license, unless indicated otherwise in a credit line to the material. If material is not included in the article's Creative Commons license and your intended use is not permitted by statutory regulation or exceeds the permitted use, you will need to obtain permission directly from the copyright holder. To view a copy of this license, visit http://creativecommons.org/licenses/by/4.0/.

(c) The Author(s) 2020 